\title{
Plasmon dispersion in layered transition-metal dichalcogenides
}

\author{
Pierluigi Cudazzo, ${ }^{1}$ Matteo Gatti, ${ }^{1}$ and Angel Rubio ${ }^{1,2}$ \\ ${ }^{1}$ Nano-Bio Spectroscopy Group and ETSF Scientific Development Centre, Departamento Física de Materiales, Universidad del País Vasco, \\ Centro de Física de Materiales CSIC-UPVIEHU-MPC and DIPC, Avenida Tolosa 72, E-20018 San Sebastián, Spain \\ ${ }^{2}$ Fritz-Haber-Institut der Max-Planck-Gesellschaft, Theory Department, Faradayweg 4-6, D-14195 Berlin-Dahlem, Germany
}

(Received 22 March 2012; revised manuscript received 12 May 2012; published 14 August 2012)

\begin{abstract}
Motivated by recent experiments, we perform a microscopic analysis of the dynamical charge response of layered transition-metal dichalcogenides that display a low-temperature charge-density wave (CDW) order. In agreement with measurements, our parameter-free results show a negative in-plane plasmon dispersion that switches to positive slope upon electron (or hole) doping. This finding is explained by the peculiar behavior of the intraband transitions, which are partially suppressed under doping, and it is not linked to the CDW order. Finally, in the direction perpendicular to the layers, we predict the reappearance around the Bragg reflections of the spectra of the first Brillouin zone, a clear effect of the crystal local-field impact. Our results give a general picture of the collective excitations in these materials suggesting a simpler reinterpretation of the experiments.

DOI: 10.1103/PhysRevB.86.075121

PACS number(s): 71.45.Lr, 71.45.Gm, 73.21.Ac, 78.70.Ck
\end{abstract}

\section{INTRODUCTION}

Layered transition-metal dichalcogenides (TMDC) ${ }^{1}$ have attracted considerable interest for the coexistence of competing electronic orders: notably, charge-density wave (CDW) and superconductivity. In particular, an intense debate has been created over the years about the origin of the CDW order. ${ }^{2}$ Several explanations have been put forward: from Fermisurface nestings ${ }^{3}$ to van Hove singularities (saddle points) in the density of states (DOS), ${ }^{4}$ until a recent theoretical work that pointed to the role of electron-phonon coupling, ruling out a pure electronic mechanism for the CDW instability. ${ }^{5}$ Moreover, similarities with cuprates, in both the competition between CDW and superconductivity and the presence of a pseudogap, have driven further interest in these compounds on a fundamental level (see for example Ref. 6).

The dynamical charge-density response is a key quantity for setting both the CDW and superconductivity orders, because it provides the renormalization of the electron-electron and electron-phonon interactions. Thus, its investigation is of the utmost importance for understanding the electronic properties of these materials. In fact, a very recent experimental study has reported an intriguing result. ${ }^{7}$ Electron energyloss spectroscopy (EELS), which measures the loss function $L(\mathbf{q}, \omega)=-\operatorname{Im} \epsilon_{M}^{-1}(\mathbf{q}, \omega) \quad\left(\epsilon_{M}\right.$ is the macroscopic dielectric function and $\mathbf{q}$ is the momentum transfer), provides a direct access to the dynamical response of the materials. EELS spectra have revealed a negative dispersion of the plasmonsthe collective charge-density modes of the material-in three prototypical TMDC $\left(2 \mathrm{H}-\mathrm{TaS}_{2}, 2 \mathrm{H}-\mathrm{TaSe}_{2}\right.$, and $\left.2 \mathrm{H}-\mathrm{NbSe}_{2}\right) .{ }^{7-9}$ This is in clear contrast with the expectation deriving from the homogeneous electron gas (HEG), where the plasmon energy, in the random-phase approximation (RPA), has a positive parabolic dispersion: $\omega_{p}(q)=\omega_{0}+\alpha q^{2}$, where $\omega_{0}$ is proportional to the square root of the carrier density and $\alpha>0$ to the square of the Fermi velocity. ${ }^{10}$ By introducing a macroscopic semiclassical Ginzburg-Landau model, it was concluded that the negative dispersion in TMDC is the consequence of the collective charge fluctuations associated to the CDW order that are capable to reverse the positive dispersion of the "bare" plasmons. ${ }^{7}$

Actually, negative plasmon dispersions have been demonstrated in several other materials: from a heavy alkali metal such as $\mathrm{Cs}^{11-13}$ to doped molecular crystals. ${ }^{14}$ In many cases, deviations from the standard HEG behavior have been reported. ${ }^{11,15-20}$ In fact, real metals are often far from a HEG model with a single band and a spherical Fermi surface. Therefore, these observations call for a microscopic analysis of the dynamical response of the real compounds, beyond a HEG model. This is especially true in materials like the TMDC under study, which display multiple narrow $d$ bands crossing the Fermi level $\left(E_{F}\right)$ with anisotropic effective masses. The question is to quantify the impact of band structure effects in the dynamical response of TMDC.

Thus, here we perform an ab initio calculation of the loss function of four TMDC (those measured in Ref. 7 plus $2 \mathrm{H}-\mathrm{NbS}_{2}$ ), by making use of parameter-free time-dependent density-functional theory (TDDFT). ${ }^{21}$ We reproduce the inplane plasmon dispersion in agreement with experiment. ${ }^{7-9}$ Our analysis shows that the unusual dispersion in TMDC is due to the peculiar behavior of the intraband transitions that contribute to build the plasmon. Hence, there is no need to invoke a coupling with the CDW order. In fact, when intraband transitions are suppressed, e.g., by electron doping, the dispersion becomes positive. Finally, we predict that the loss function in the direction perpendicular to the layers displays the reappearance around the Bragg reflections of the spectra of the first Brillouin zone, a manifestation of the coupling between excitations at small and large momentum transfers. ${ }^{18,22}$

\section{RESULTS AND DISCUSSION}

The microscopic dielectric function $\epsilon$ is directly related to the susceptibility $\chi:{ }^{23} \epsilon^{-1}=1+v \chi$ ( $v$ is the Coulomb potential). In TDDFT, $\chi$ is the solution of a Dyson-like equation: $\chi=\chi_{0}+\chi_{0}\left(v+f_{x c}\right) \chi$, where $\chi_{0}$ is the Kohn-Sham (KS) susceptibility and $f_{x c}$ is the nonlocal frequency-dependent 


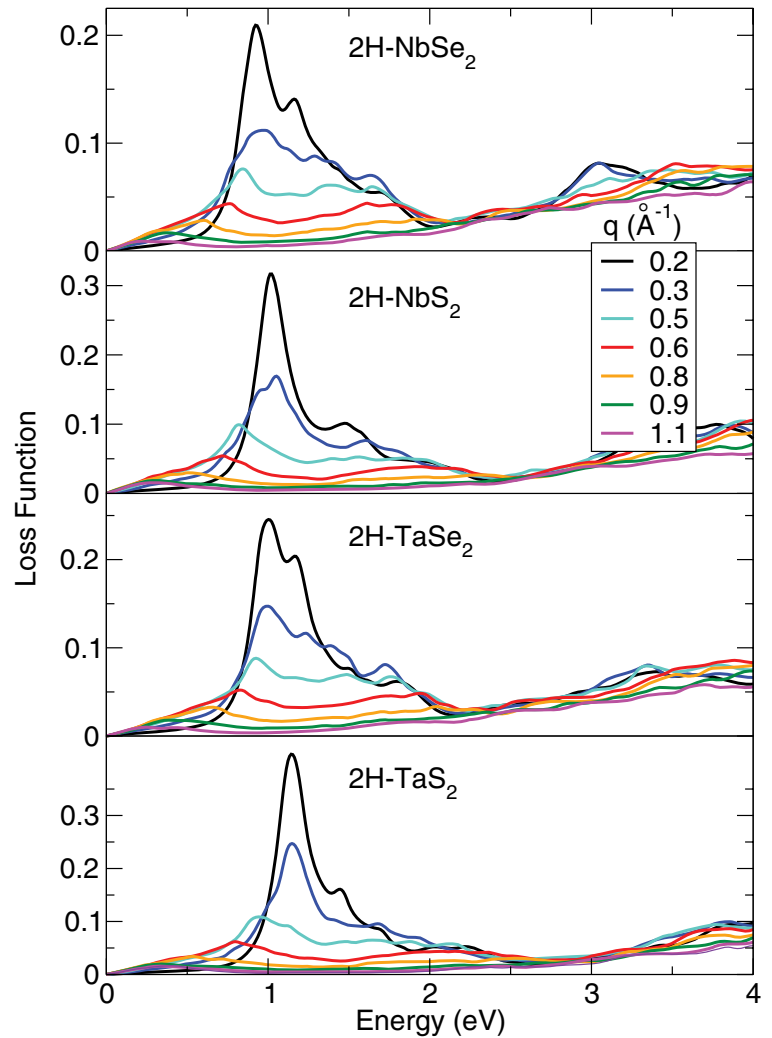

FIG. 1. (Color online) Calculated loss functions of the four TMDC calculated for momentum transfers along the in-plane $\Gamma K$ direction.

exchange-correlation kernel, for which we use the adiabatic local-density approximation (ALDA). When $f_{x c}=0$ the usual RPA is retrieved. All these microscopic quantities in a solid are matrices in reciprocal lattice vectors $\mathbf{G}$. The macroscopic dielectric function $\epsilon_{M}$ is given by $\epsilon_{M}(\mathbf{q}, \omega)=1 / \epsilon_{\mathbf{G}, \mathbf{G}}^{-1}\left(\mathbf{q}_{r}, \omega\right)$, where $\mathbf{q}=\mathbf{q}_{r}+\mathbf{G}$ and $\mathbf{q}_{r}$ belongs to the first Brillouin zone. The loss function $L(\mathbf{q}, \omega)$ is $-\operatorname{Im} \epsilon_{M}^{-1}(\mathbf{q}, \omega)$.

In Fig. 1 we show the loss function of the TMDC calculated in RPA for different momentum transfers $\mathbf{q}$ along the $\Gamma K$ direction. ${ }^{24}$ The main peak in the spectra at $\omega \sim 0.9-1.1 \mathrm{eV}$ (at $q=0.2 \AA^{-1}$ ) is related to a zero of the real part of the dielectric function $\epsilon_{1}=\operatorname{Re} \epsilon_{M}$. Thus it is a plasmon. From our analysis, we find that it is due to excitations between the two narrow $d$ bands that cross $E_{F}$ (in the following, we will call them "intraband transitions"), which give rise to a peak in $\epsilon_{2}=\operatorname{Im} \epsilon_{M}$ at $\omega \sim 0.30-0.38 \mathrm{eV}$ with $q=0.2 \AA^{-1}$. The band structure is similar in all the TMDC (see Appendix A). There is also a third dispersive $p_{z}$ band that does not contribute to this in-plane plasmon.

In all the TMDC, by increasing $q$, we observe a negative shift of the plasmon peak, which looses intensity and becomes broader, entering the particle-hole continuum. Those results are in very good agreement with the recent experiments, ${ }^{7-9}$ in both the position and the shape of the spectral structures, except for $2 \mathrm{H}-\mathrm{NbS}_{2}$ where an old experiment ${ }^{29}$ measured a positive plasmon dispersion (we will set back to this point below). We can now address in detail the issue of understanding the origin of the negative dispersion of this intraband plasmon.
Several possibilities to explain deviations from the HEG RPA result have been put forward in the past for different materials. Increasing the momentum transfer $q$ may induce the activation of dipole-forbidden interband transitions above the plasmon energy, which in turn shifts to lower energies the zero crossing of $\epsilon_{1}$ and hence the plasmon. ${ }^{12}$ Also crystal local-field effects (LFE), ${ }^{23}$ which are related to induced spatial charge fluctuations (quantified by the off-diagonal components of $\epsilon$ ), may alter considerably the plasmon dispersion in inhomogeneous materials. ${ }^{15-18}$ Finally, correlation effects beyond RPA (i.e., for $f_{x c} \neq 0$ ) tend to reduce the plasmon dispersion. ${ }^{13}$ However, none of those effects is actually at play in the TMDC. Increasing $q$, no new peaks above the plasmon energy appear in the spectra, LFE are not relevant for in-plane $q$ (within the layers, TMDC are pretty homogeneous ${ }^{15}$ ), and also the ALDA results (see Appendix B) are very similar to RPA.

From all these observations it would seem that the $d$ electrons that give rise to the plasmon in the TMDC behave like a HEG. However, contrary to the HEG, the $d$ bands are narrow and not parabolic. We now show how the peculiar properties of those bands are rightly at the origin of the negative plasmon dispersion. By using the Kramers-Kronig relations, we introduce ${ }^{12,14}$

$$
\begin{aligned}
& D_{-}(\mathbf{q}, \omega)=\frac{2}{\pi} \int_{0}^{\omega} d \omega^{\prime} \frac{\omega^{\prime} \epsilon_{2}\left(\mathbf{q}, \omega^{\prime}\right)}{\omega^{2}-\omega^{\prime 2}}, \\
& D_{+}(\mathbf{q}, \omega)=\frac{2}{\pi} \int_{\omega}^{\infty} d \omega^{\prime} \frac{\omega^{\prime} \epsilon_{2}\left(\mathbf{q}, \omega^{\prime}\right)}{\omega^{\prime 2}-\omega^{2}} .
\end{aligned}
$$

In the present case, the $D_{-}$term corresponds to intraband transitions and the $D_{+}$term to interband transitions. The frequencies $\omega_{p}=\omega_{p}(\mathbf{q})$ for which $\epsilon_{1}\left(\mathbf{q}, \omega_{p}\right)=1-D_{-}\left(\mathbf{q}, \omega_{p}\right)+$ $D_{+}\left(\mathbf{q}, \omega_{p}\right)=0$ define the plasmon dispersion. In the absence of interband transitions above $\omega_{p}$ (like in the HEG), $D_{+}\left(\mathbf{q}, \omega_{p}\right)=0$. Moreover, in the HEG $\epsilon_{2}$ is proportional to the joint density of states (JDOS): $\epsilon_{2}(\mathbf{q}, \omega) \propto \operatorname{JDOS}(\mathbf{q}, \omega) /|\mathbf{q}|^{2}$. The presence of a wide parabolic energy band gives rise to a sharp peak in the JDOS with strong positive dispersion with $q$ [see Fig. 2(a)]. In turn, $D_{-}\left(\mathbf{q}, \omega_{p}\right)$ is an increasing function of $q$, and the zeros of $\epsilon_{1}=1-D_{-}$are shifted to higher frequencies progressively with $q\left(\epsilon_{1}<0\right.$ for $\left.\omega \lesssim \omega_{p}\right)$. The plasmon has a positive dispersion. A completely different behavior is found in the TMDC. The narrow $d$ bands, which are flat in a wide region of the first Brillouin zone (see Appendix A), give rise to an intense and nondispersive peak in the JDOS [see Fig. 2(a)]. As a consequence, the $D_{-}\left(\mathbf{q}, \omega_{p}\right)$ term is a decreasing function of $q$. Therefore the plasmon dispersion is negative. In fact, the consideration of oscillator strength matrix elements (ME) in the calculation of $\epsilon$ does not affect this behavior. The ME have a weak positive dispersion and decrease in amplitude with $q$. Finally, higher energy interband transitions, contained in the $D_{+}\left(\mathbf{q}, \omega_{p}\right)$ term in Eq. (1), screen the intraband plasmon. This has two main effects: (i) a redshift of the plasmon frequency and (ii) a reduction of the negative dispersion due to the fact that the screening becomes weaker as $q$ increases [i.e., $D_{+}\left(\mathbf{q}, \omega_{p}\right)$ is a decreasing function of $\left.q\right]$. Thus, interband transitions act to suppress the negative dispersion and switch it to a positive slope. However, in this case, due to the large intraband peak in $\epsilon_{2}$, interband transitions do not give a remarkable contribution and the intrinsic negative plasmon 

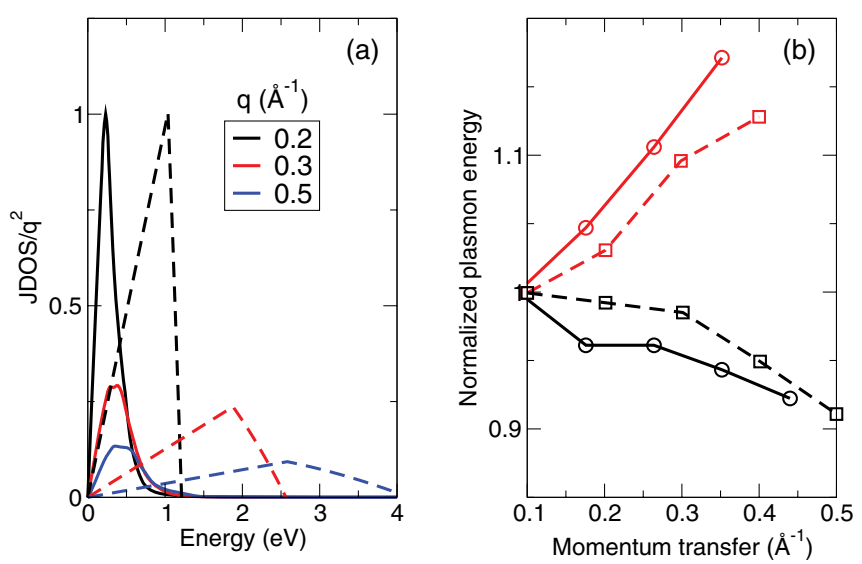

FIG. 2. (Color online) (a) Joint density of states $\operatorname{JDOS}(\mathbf{q}, \omega) /|\mathbf{q}|^{2}$ for $2 \mathrm{H}-\mathrm{NbSe}_{2}$ (solid lines) from intraband transitions between $d$ bands and for an equivalent electron number for the HEG $\left(r_{s}=3.73\right)$ (dashed lines). Both are normalized to the intensity of the respective highest peak. The JDOS of the HEG is dispersing with $q$, while for $2 \mathrm{H}-\mathrm{NbSe}_{2}$ it is not. (b) Plasmon dispersion along $\Gamma M$ in $2 \mathrm{H}-\mathrm{TaSe}_{2}$ (black lines) and potassium intercalated $2 \mathrm{H}-\mathrm{K}_{0.64} \mathrm{TaSe}_{2}$ (red lines), normalized to the onset value at $q=0.1 \AA^{-1}$. Results of our calculations (solid lines) are compared with experimental data (dashed lines) from Ref. 8. The negative dispersion is switched to positive upon doping. A similar qualitative picture holds for the other three TMDC studied in this work.

dispersion is preserved. The present analysis is general and shows that the negative plasmon dispersion is a basic property of the TMDC, directly related to the shape of the bands crossing $E_{F}$.

To corroborate our analysis, we simulate electron doping of $2 \mathrm{H}-\mathrm{TaSe}_{2}$ by shifting upwards the Fermi level, according to a simple rigid-band model. In this way, previously empty bands are populated, suppressing intraband transitions. Due to its intraband origin, electron (or hole) doping decreases the plasmon energy. ${ }^{30}$ More important for the present discussion is that under doping the plasmon dispersion is switched to a positive slope [see Fig. 2(b)]. In fact, under doping the spectral weight in $\epsilon_{2}$ is transferred from the intraband towards the interband transitions, so that the interband contribution becomes strong enough to completely suppress the negative dispersion of the intraband plasmon. This result is in agreement with recent measurements on potassium-intercalated $2 \mathrm{H}-\mathrm{TaSe}_{2} .{ }^{8}$ Also in the real doped material the plasmon dispersion becomes positive in agreement with our calculations [see Fig. 2(b)]. Moreover, this provides a hint to explain the observed positive dispersion in $2 \mathrm{H}-\mathrm{NbS}_{2}{ }^{29}$ In that case, the sample could have been not perfectly stoichiometric (in fact, $2 \mathrm{H}-\mathrm{NbS}_{2}$ is unstable during the process growth; see Ref. 8, p. 82).

Instead, rotating the momentum transfer $\mathbf{q}$ to be perpendicular to the TMDC planes (i.e., along the $\Gamma A$ direction), we can now predict a different behavior (which has not been measured yet). Here we just consider $2 \mathrm{H}-\mathrm{NbSe}_{2}$, but the other TMDC are similar. We find three main structures in the spectra calculated including crystal local fields (A, B, and C in the right panel of Fig. 3).

We first discuss the structures $\mathrm{A}$ and $\mathrm{B}$, which are very similar in the calculations with or without local fields (left

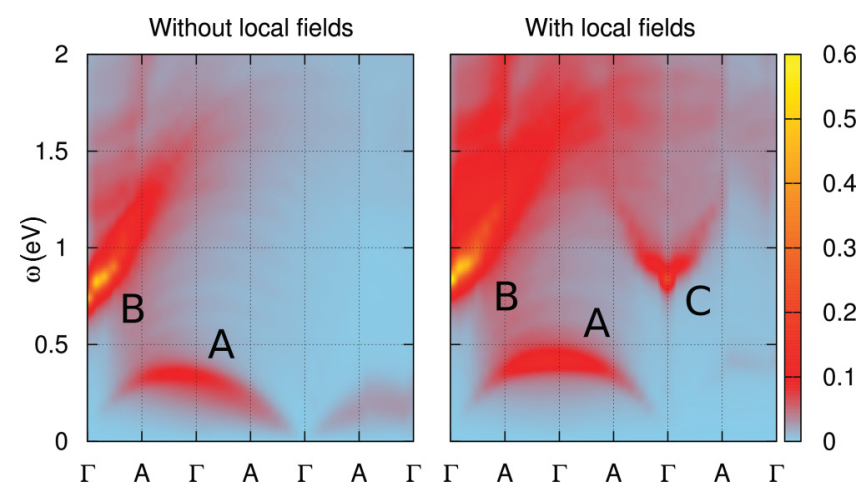

FIG. 3. (Color online) Loss function of $2 \mathrm{H}-\mathrm{NbSe}_{2}$ along the $\Gamma A$ direction, perpendicular to the layers, up to the 4th Brillouin zone, calculated without and with LFE (left and right panels, respectively). The labels A, B, and C mark the main structures in the spectra as discussed in the main text.

panel of Fig. 3). ${ }^{31}$ Both structures are plasmon excitations, related to zeros (or minima) of $\epsilon_{1}$, located at energies around $0.16 \mathrm{eV}$ (structure A) and $0.89 \mathrm{eV}$ (structure B) for $q=$ $0.04 \AA^{-1}$. The structure A derives from $d \rightarrow d$ transitions (with a peak in $\epsilon_{2}$ at $0.05 \mathrm{eV}$ ), and the structure $\mathrm{B}$ from $p_{z} \rightarrow p_{z}$ and $p_{z} \rightarrow d$ transitions at $0.2 \mathrm{eV}$. Thus, we call " $d$ plasmon" the former and " $p+d$ plasmon" the latter. Contrary to the in-plane direction, here both $d$ and $p+d$ plasmons show a positive dispersion along $\Gamma A$. In particular, in the absence of interband transitions the $p+d$ plasmon has a negative dispersion, since also in this case the JDOS does not disperse. However, screening effects arising from interband transitions above the plasmon frequency are strong enough to change its slope. The $d$ plasmon has the same nature of the in-plane plasmon. However, now it is strongly screened by the $p+d$ plasmon. In fact, its positive dispersion and its increase in intensity at small $q$ are related to the interference with $p_{z} \rightarrow d$ transitions, which are absent for in-plane momentum transfer. Interestingly, at higher $q$ crossing the second Brillouin zone, the dispersion of the $d$ plasmon turns its slope becoming negative. This result is due to the behavior of the ME of $d \rightarrow d$ transitions, which draw back the imaginary part of the dielectric function.

The third peak (structure $\mathrm{C}$ in right panel of Fig. 3) appears at momentum transfers in the third Brillouin zone at the same energy of the $p+d$ plasmon (structure B), only when LFE are taken into account. A similar effect has been found and discussed in the case of graphite. ${ }^{18,22}$ Thus here we can follow that analysis. We write the dielectric function at $\mathbf{q}=\mathbf{q}_{r}+$ $\mathbf{G}_{0}$ with small $\mathbf{q}_{r}$ in a $2 \times 2$ matrix approximation, similarly to the two-plasmon-band model ${ }^{33}$ [i.e., we assume that all off-diagonal matrix elements $\epsilon_{\mathbf{G}, \mathbf{G}^{\prime}}\left(\mathbf{q}_{r}, \omega\right)$ are negligible with respect to $\left.\epsilon_{\mathbf{0}, \mathbf{G}_{0}}\left(\mathbf{q}_{r}, \omega\right)\right]$ :

$$
\begin{aligned}
\epsilon_{\mathbf{G}_{0}, \mathbf{G}_{0}}^{-1}\left(\mathbf{q}_{r}, \omega\right)= & \frac{1}{\epsilon_{\mathbf{G}_{0}, \mathbf{G}_{0}}\left(\mathbf{q}_{r}, \omega\right)} \\
& +\frac{\epsilon_{\mathbf{G}_{0} \mathbf{0}}\left(\mathbf{q}_{r}, \omega\right) \epsilon_{\mathbf{0}, \mathbf{G}_{0}}\left(\mathbf{q}_{r}, \omega\right)}{\left[\epsilon_{\mathbf{G}_{0}, \mathbf{G}_{0}}\left(\mathbf{q}_{r}, \omega\right)\right]^{2}} \epsilon_{\mathbf{0 , 0}}^{-1}\left(\mathbf{q}_{r}, \omega\right) .
\end{aligned}
$$

The first term in Eq. (2) is the result that we would obtain neglecting LFE and represents the contribution arising from 

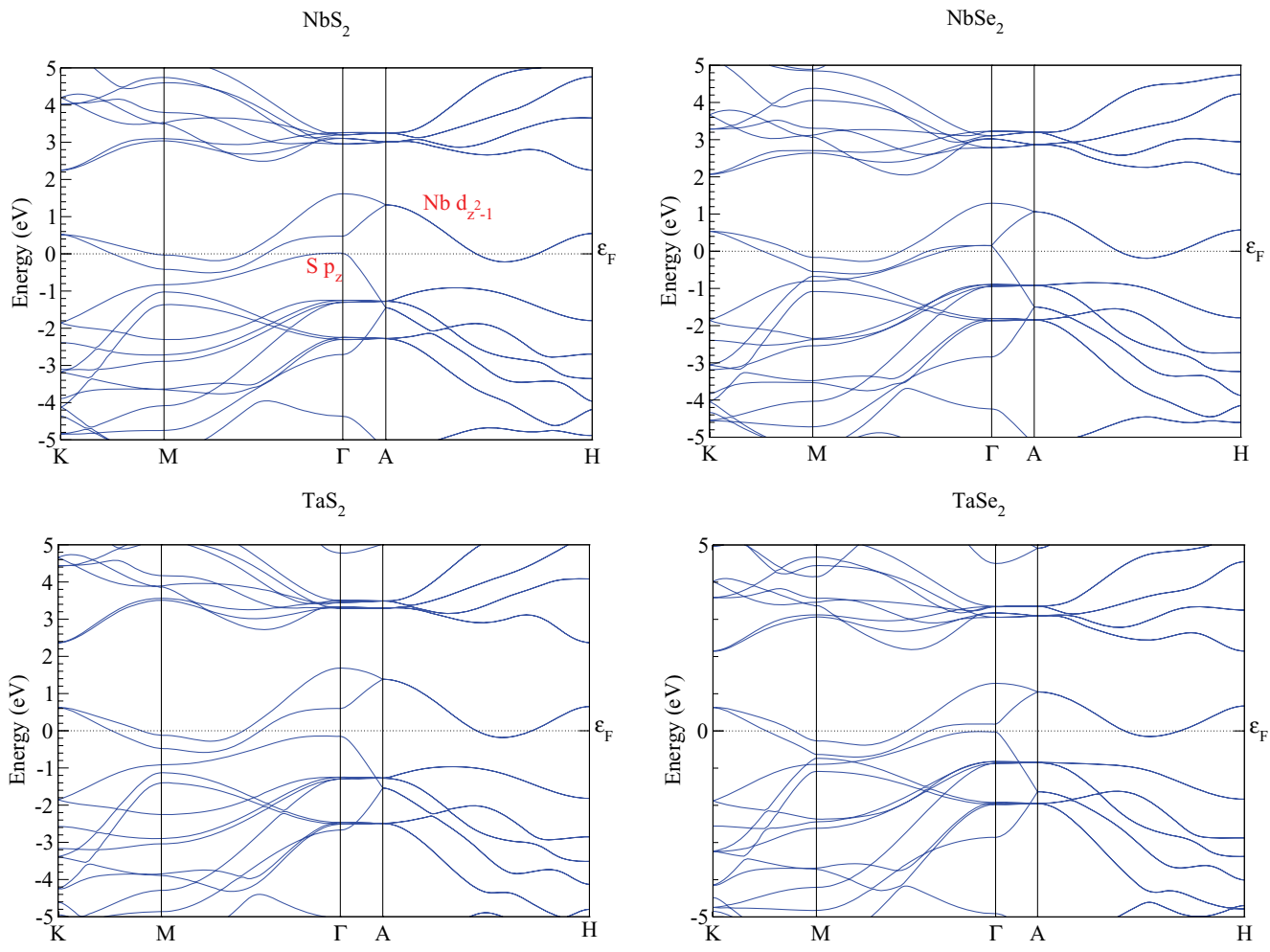

FIG. 4. (Color online) Band structure of the four transition-metal dichalcogenides studied in this work. The labels in the top-left panel indicate the character (at the $\Gamma$ point) of the electronic states that give rise to the plasmon (analogously for the other materials).

independent electron-hole excitations with high momentum transfer, being the $p+d$ plasmon completely suppressed in the higher Brillouin zones (see left panel of Fig. 3). The second term, on the other hand, is related to the dielectric function evaluated in the first Brillouin zone (where the spectrum is dominated by the $p+d$ plasmon) and causes the reappearance of the spectrum $\epsilon_{\mathbf{0}, \mathbf{0}}^{-1}\left(\mathbf{q}_{r}, \omega\right)$, weighted by a coupling term related to the off-diagonal part of the dielectric matrix. Thus, from Eq. (2) the effect of crystal local fields is clear: They are responsible for the coupling between the $p+d$ plasmon at $\mathbf{q}=$ $\mathbf{q}_{r}$ in the first Brillouin zone and the independent electron-hole excitations with momentum transfer $\mathbf{q}=\mathbf{q}_{r}+\mathbf{G}_{0}$ belonging to the higher Brillouin zone identified by $\mathbf{G}_{0}$. If LFE are strong enough and the coupling term has a weak energy dependence, the second term becomes dominant and the spectrum at small momentum transfer reappears in higher Brillouin zones. ${ }^{16,18,22,34}$ This is just what happens in TMDC when $\mathbf{G}_{0}=(0,0,2)$ (third Brillouin zone). In fact $\epsilon_{\mathbf{G}_{0}, \mathbf{G}_{0}}^{-1}\left(\mathbf{q}_{r}, \omega\right)$ is very similar, up to a renormalization constant, to $\epsilon_{\mathbf{0}, \mathbf{0}}^{-1}\left(\mathbf{q}_{r}, \omega\right)$. The small difference between the two spectra arises from the weak frequency dependence of the coupling term and from the contribution of the other off-diagonal matrix elements neglected in Eq. (2). Moreover, the coupling term in Eq. (2) is zero when $\mathbf{G}_{0}$ identifies a plane in the reciprocal space where the Bragg reflection is forbidden. ${ }^{18,22}$ In the TMDC, Bragg reflections along the $z$ axis are allowed only when $\mathbf{G}_{0}=(0,0,2 m)$ (with $m$ integer). Thus, in the second Brillouin zone when $\mathbf{G}_{0}=(0,0,1)$, the coupling term in Eq. (2) is zero and LFE derive only from the small contribution of the other off-diagonal matrix elements. In fact, the spectrum in the second Brillouin zone around $\mathbf{G}_{0}=(0,0,1)$ is similar to that obtained without LFE.

\section{CONCLUSIONS}

In conclusion, the onset of the CDW order at low temperature is known to affect the dynamical response of the TMDC. By changing the underlying band structure, new electron-hole transitions appear, leading to an antiscreening of the plasmon, ${ }^{30}$ and a concomitant increase of its bandwidth. ${ }^{7-9}$ However, our $a b$ initio analysis has shown that the origin of the measured negative in-plane plasmon dispersion in the disordered phase of TMDC stems from the peculiar behavior of the intraband transitions that give rise to the plasmon. Therefore, there is no need to invoke an effect of the CDW to explain it. This has allowed us to understand also why under doping the slope of the plasmon dispersion is switched to positive, which is verified experimentally by intercalating $2 \mathrm{H}-\mathrm{TaSe}_{2}$ with $\mathrm{K} .{ }^{8}$ Finally, we have predicted the plasmon dispersion along the direction perpendicular to the layers, with the reappearance around Bragg reflections of the plasmon of the first Brillouin zone. Our analysis is very general and allows a simpler reinterpretation of the experiments. We expect to find similar results in other TMDC with narrow bands crossing $E_{F}$. Thus it represents a step forward in the understanding of this challenging class of materials. In fact, the dynamical response and the screening of the Coulomb interaction are essential for describing photoemission spectra and the electron-electron and electron-phonon renormalizations for the onset of CDW and superconductivity. 


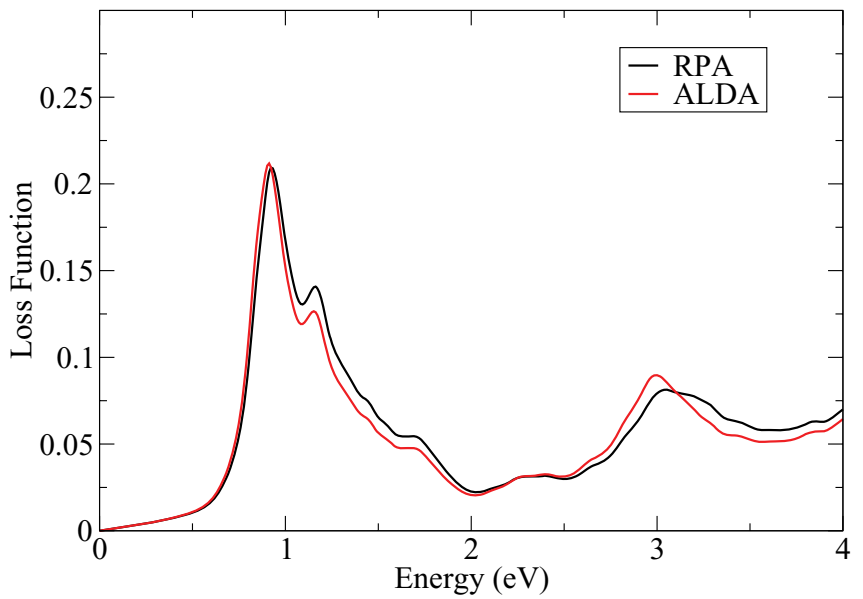

FIG. 5. (Color online) Loss function of $\mathrm{NbSe}_{2}$ at $q=0.2 \AA^{-1}$ along the $\Gamma K$ line evaluated in two different approximation: RPA and ALDA.

\section{ACKNOWLEDGMENTS}

Financial support was provided by Spanish (FIS201165702-C02-01 and PIB2010US-00652), ACI-Promociona
(ACI2009-1036), and Grupos Consolidados UPV/EHU del Gobierno Vasco (IT-319-07) grants and the European Research Council Advanced Grant DYNamo (ERC-2010-AdG, Proposal No. 267374). Computational time was granted by i2basque and BSC "Red Española de Supercomputacion."

\section{APPENDIX A: BAND STRUCTURES}

In Fig. 4 we show the band structures calculated in LDA for $\mathrm{NbS}_{2}, \mathrm{NbSe}_{2}, \mathrm{TaS}_{2}$, and $\mathrm{TaSe}_{2}$. They are very similar in all the four cases. We also indicate the three bands around the Fermi energy that determine the low-energy dynamical response of the four materials, as discussed in the previous sections (see also Ref. 5).

\section{APPENDIX B: ALDA RESULTS}

In Fig. 5 we compare the loss function calculated in RPA and ALDA for $\mathrm{NbSe}_{2}$. We see that the two results are very similar, showing that exchange-correlation effects beyond RPA are not changing the conclusions discussed in the previous sections.
${ }^{1}$ J. A. Wilson and A. D. Yoffe, Adv. Phys. 18, 193 (1969).

${ }^{2}$ For a recent review see, e.g., K. Rossnagel, J. Phys.: Condens. Matter 23, 213001 (2011)

${ }^{3}$ J. A. Wilson, F. J. Di Salvo, and S. Mahajan, Phys. Rev. Lett. 32, 882 (1974).

${ }^{4}$ T. M. Rice and G. K. Scott, Phys. Rev. Lett. 35, 120 (1975).

${ }^{5}$ M. D. Johannes, I. I. Mazin, and C. A. Howells, Phys. Rev. B 73, 205102 (2006); M. D. Johannes and I. I. Mazin, ibid. 77, 165135 (2008).

${ }^{6}$ T. Kiss, T. Yokoya, A. Chainani, S. Shin, T. Hanaguri, M. Nohara, and H. Takagi, Nat. Phys. 3, 720 (2007); S. V. Borisenko, A. A. Kordyuk, A. N. Yaresko, V. B. Zabolotnyy, D. S. Inosov, R. Schuster, B. Büchner, R. Weber, R. Follath, L. Patthey, and H. Berger, Phys. Rev. Lett. 100, 196402 (2008); S. V. Borisenko, A. A. Kordyuk, V. B. Zabolotnyy, D. S. Inosov, D. Evtushinsky, B. Büchner, A. N. Yaresko, A. Varykhalov, R. Follath, W. Eberhardt, L. Patthey, and H. Berger, ibid. 102, 166402 (2009).

${ }^{7}$ J. van Wezel, R. Schuster, A. König, M. Knupfer, J. van den Brink, H. Berger, and B. Büchner, Phys. Rev. Lett. 107, 176404 (2011).

${ }^{8}$ R. Schuster, Ph.D. thesis, Technische Universität Dresden (Germany), 2009, http://nbn-resolving.de/urn:nbn:de:bsz:14qucosa-27333.

${ }^{9}$ R. Schuster, R. Kraus, M. Knupfer, H. Berger, and B. Büchner, Phys. Rev. B 79, 045134 (2009).

${ }^{10}$ G. F. Giuliani and G. Vignale, Quantum Theory of the Electron Liquid (Cambridge University Press, Cambridge, 2005).

${ }^{11}$ A. vom Felde, J. Sprösser-Prou, and J. Fink, Phys. Rev. B 40, 10181 (1989).

${ }^{12}$ F. Aryasetiawan and K. Karlsson, Phys. Rev. Lett. 73, 1679 (1994).

${ }^{13}$ A. Fleszar, R. Stumpf, and A. G. Eguiluz, Phys. Rev. B 55, 2068 (1997).

${ }^{14}$ P. Cudazzo, M. Gatti, F. Roth, B. Mahns, M. Knupfer, and A. Rubio, Phys. Rev. B 84, 155118 (2011).
${ }^{15}$ A. G. Marinopoulos, L. Reining, V. Olevano, A. Rubio, T. Pichler, X. Liu, M. Knupfer, and J. Fink, Phys. Rev. Lett. 89, 076402 (2002); A. G. Marinopoulos, L. Reining, A. Rubio, and V. Olevano, Phys. Rev. B 69, 245419 (2004).

${ }^{16}$ Y. Q. Cai, P. C. Chow, O. D. Restrepo, Y. Takano, K. Togano, H. Kito, H. Ishii, C. C. Chen, K. S. Liang, C. T. Chen, S. Tsuda, S. Shin, C. C. Kao, W. Ku, and A. G. Eguiluz, Phys. Rev. Lett. 97 176402 (2006)

${ }^{17}$ C. Kramberger, R. Hambach, C. Giorgetti, M. H. Rümmeli, M. Knupfer, J. Fink, B. Büchner, L. Reining, E. Einarsson, S. Maruyama, F. Sottile, K. Hannewald, V. Olevano, A. G. Marinopoulos, and T. Pichler, Phys. Rev. Lett. 100, 196803 (2008).

${ }^{18}$ R. Hambach, C. Giorgetti, F. Sottile, L. Reining, N. Hiraoka, Y. Q. Cai, A. G. Marinopoulos, and F. Bechstedt, Phys. Rev. Lett. 101, 266406 (2008)

${ }^{19}$ S. Huotari, C. Sternemann, M. C. Troparevsky, A. G. Eguiluz, M. Volmer, H. Sternemann, H. Müller, G. Monaco, and W. Schülke, Phys. Rev. B 80, 155107 (2009).

${ }^{20}$ S. Huotari, M. Cazzaniga, H.-Ch. Weissker, T. Pylkkänen, H. Müller, L. Reining, G. Onida, and G. Monaco, Phys. Rev. B 84, 075108 (2011); M. Cazzaniga, H.-Ch. Weissker, S. Huotari, T. Pylkkänen, P. Salvestrini, G. Monaco, G. Onida, and L. Reining, ibid. 84, 075109 (2011)

${ }^{21}$ E. Runge and E. K. U. Gross, Phys. Rev. Lett. 52, 997 (1984).

${ }^{22}$ R. Hambach, Ph.D. thesis, Ecole Polytechnique, Palaiseau (France), 2010, http://etsf.polytechnique.fr/system/files/PhD_Hambach_ 2010.pdf.

${ }^{23}$ For a review of the theoretical framework: G. Onida, L. Reining, and A. Rubio, Rev. Mod. Phys. 74, 601 (2002).

${ }^{24}$ In our calculations, we adopt the experimental lattice structures (Ref. 25) and use norm-conserving pseudopotentials in a planewave expansion (with an energy cutoff between 30 and $40 \mathrm{Ha}$ ) 
(Refs. 26 and 27). We use a $24 \times 24 \times 12$ grid of $\mathbf{k}$ points and include 100 bands in the calculation. The dimension of the matrices is $300 \mathbf{G}$ vectors. With those parameters we get converged results in the range $0-10 \mathrm{eV}$. Spin-orbit coupling is known to induce visible effects in the band structures (see, e.g., Refs. 5 and 28). However, its inclusion in the calculation would not affect our conclusions.

${ }^{25}$ D. E. Moncton, J. D. Axe, and F. J. Di Salvo, Phys. Rev. B 16, 801 (1977); W. G. Fisher and M. J. Sienko, Inorg. Chem. 19, 39 (1980); A. Meetsma, G. A. Wiegers, R. J. Haange, and J. L. de Boer, Acta Crystallogr. Sect. C 46, 1598 (1990).

${ }^{26}$ X. Gonze, G.-M. Rignanese, M. Verstraete, J.-M. Beuken, Y. Pouillon, R. Caracas, F. Jollet, M. Torrent, G. Zerah, M. Mikami, Ph. Ghosez, M. Veithen, J.-Y. Raty, V. Olevano, F. Bruneval, L. Reining, R. Godby, G. Onida, D. R. Hamann, and D. C. Allan, Z. Kristallogr. 220, 558 (2005).
${ }^{27}$ A. Marini, C. Hogan, M. Gruning, and D. Varsano, Comput. Phys. Commun. 180, 1392 (2009).

${ }^{28}$ S. V. Borisenko, A. A. Kordyuk, V. B. Zabolotnyy, D. V. Evtushinsky, T. K. Kim, B. Büchner, A. N. Yaresko, V. D. Borisenko, and H. Berger, J. Phys. Chem. Solids 72, 562 (2011).

${ }^{29}$ R. Manzke, G. Crecelius, J. Fink, and R. Schöllhorn, Solid State Commun. 40, 103 (1981).

${ }^{30}$ G. Campagnoli, A. Gustinetti, A. Stella, and E. Tosatti, Phys. Rev. B 20, 2217 (1979).

${ }^{31}$ Following Ref. 32, we have extrapolated the intraband contribution for $\mathbf{q} \rightarrow 0$, giving rise to a Drude plasmon $\omega_{D}=(2.13,0.85) \mathrm{eV}$.

${ }^{32}$ A. Marini, G. Onida, and R. Del Sole, Phys. Rev. B 64, 195125 (2001).

${ }^{33}$ L. E. Oliveira and K. Sturm, Phys. Rev. B 22, 6283 (1980).

${ }^{34}$ K. Sturm, W. Schülke, and J. R. Schmitz, Phys. Rev. Lett. 68, 228 (1992). 